\title{
Age-Dependency of Clinical Characteristics of Patients Participating Cardiovascular Rehabilitation Results from the German Registry of Ambulatory Cardiac Rehabilitation-KARREE
}

\author{
Roland Nebel1* ${ }^{*}$, Michael Marx², Michael Geier ${ }^{3}$, Brigitte Buran-Kilian4, \\ Taoufik Ouarrak ${ }^{5}$, Manju Guha ${ }^{6}$, Gregor Sauer ${ }^{7 \#, ~ G e r d ~ B o ̈ n n e r ~}{ }^{8}$, Harry Hahmann', \\ Ralf Jordan 10, Michaela Julia Engelhard11, Bernhard Rauch5 ${ }^{5}$ Birna Bjarnason-Wehrens ${ }^{11}$ \\ ${ }^{1}$ Medicos.Osnabrück GmbH, Osnabrück, Germany \\ ${ }^{2}$ Zentrum für Gefäßmedizin Bonn, KaRO GmbH Waldbröl, Bonn, Germany \\ ${ }^{3}$ Ambulante Rehabilitation für Herzpatienten, Evangelisches Krankenhaus Bergisch Gladbach, \\ Bergisch Gladbach, Germany \\ ${ }^{4}$ SchwerpunktReha, Ambulante kardiologische Rehabilitation, Bonn, Germany \\ ${ }^{5}$ Stiftung Institut für Herzinfarktforschung, Ludwigshafen, Germany \\ ${ }^{6}$ Reha-Klinik am Sendesaal, Bremen, Germany \\ ${ }^{7}$ Helios Rhein Klinik, Duisburg, Germany \\ ${ }^{8}$ Freiburg im Breisgau, Germany \\ ${ }^{9}$ Klinik Schwabenland, Isny, Germany \\ ${ }^{10}$ Kardiologische Gemeinschaftspraxis an der Rheinklinik, Duisburg, Germany \\ ${ }^{11}$ Deutsche Sporthochschule Köln, Cologne, Germany \\ Email: ${ }^{*}$ r.nebel@medicos-osnabrueck.de
}

Received 25 August 2014; revised 26 September 2014; accepted 28 October 2014

Copyright (C) 2014 by authors and Scientific Research Publishing Inc.

This work is licensed under the Creative Commons Attribution International License (CC BY).

http://creativecommons.org/licenses/by/4.0/

(c) (7)

\section{Abstract}

Background: Cardiovascular rehabilitation in Germany traditionally is offered as in-patient service often located far from patient's residence, and ambulatory rehabilitation still represents a minority. The German Registry of Ambulatory Cardiac Rehabilitation (KARREE) was designed to contribute to rehabilitation quality assurance and to evaluate clinical characteristics of patients participating in ambulatory rehabilitation centers. Methods: In four ambulatory rehabilitation centers 2989 patients were consecutively registered from 2008 to 2011 and evaluated with re-

\footnotetext{
*Corresponding author.

"Unfortunately, this author has been deceased.
}

How to cite this paper: Nebel, R., et al. (2014) Age-Dependency of Clinical Characteristics of Patients Participating Cardiovascular Rehabilitation Results from the German Registry of Ambulatory Cardiac Rehabilitation-KARREE. Open Journal of Therapy and Rehabilitation, 2, 207-216. http://dx.doi.org/10.4236/ojtr.2014.24026 
spect to social status, cardiovascular diagnoses and risk factors, psychological status, medication and short term clinical and social outcome. Results: Most patients referred to the ambulatory cardiac rehabilitation had an acute cardiovascular event, with patients after acute coronary syndrome representing the majority (59.9\%). Female were strongly underrepresented (16.7\%). Patient's clinical characteristics varied with the age groups evaluated $(<50$ years, $50-70$ years, $>70$ years). Whereas the reported physical inactivity, overweight and cigarette smoking was declining with age, diabetes and hypertension significantly increased. Furthermore the reported and evaluated psychosocial stress was declining with age. Regarding the group of patients still employed, $43.5 \%$ were estimated as fit for work directly at the end of the rehabilitation program, whereas a stepwise reintegration into employment was performed in $16.7 \%$. The majority of patients were transferred to ambulatory heart groups or other forms of after care for stabilizing regular physical activity. Conclusions: The large variation of the characteristics of patients participating cardiac rehabilitation underscore the need of an individualized approach for a successful implementation of secondary prevention and reintegration of these patients into their social life.

\section{Keywords}

Ambulatory Cardiac Rehabilitation, Cardiovascular Risk Factors, Acute Coronary Syndrome, Coronary Bypass Surgery, Secondary Prevention, Social Reintegration

\section{Introduction}

Cardiovascular rehabilitation (CR) in Germany is controlled by a quality assurance program, which has been developed by the German pension funds (Deutsche Rentenversicherung, DRV) in the early nineties primarily concentrating on the structures and processes of the rehabilitation centers. Some years later the quantity and intensity of the multidisciplinary therapeutic interventions during CR were evaluated in addition and included in a benchmarking process [1] [2]. Along with the implementation of this quality assurance program CR in Germany increasingly gained scientific interest by evaluating its short- and long-term effects on clinical outcome and secondary prevention measures [3]-[9]. However, as a result of the long tradition of rehabilitation clinics in Germany, these studies primarily evaluated inpatient CR. It was not before 1992 when the first model of a German ambulatory CR center was established at the Sporthochschule Köln [10]. This was followed by a variety of additional ambulatory CR projects [11]-[13]. In the meantime, ambulatory CR in Germany is officially supported by law and included into clinical routine. To guarantee minimal standards in equipment, staff and processes of ambulatory CR centers guidelines have been developed by the "Bundesarbeitsgemeinschaft für Rehabilitation" (BAR) [14]. Due to the late development of ambulatory CR in Germany there was an additional need to scientifically evaluate this setting. Several smaller studies investigating a variety of characteristics and outcomes showed non inferiority of ambulatory CR in comparison to inpatients CR [15]-[18]. The purpose of the KARREE-registry was to describe patient's characteristics and short-term clinical outcome during ambulatory CR in a large cohort of patients under all day conditions. Moreover, the special aim of this registry was to evaluate potential gender differences, and to characterize different age groups, who may need an individually adjusted rehabilitation care.

\section{Methods}

From 2008 until 2011 medical data of 2989 patients participating CR in four German ambulatory cardiovascular rehabilitation centers were consecutively evaluated for quality assurance evaluation.

CR-programs were standardized by following the BAR-guidelines and included $15 \pm 2.9$ active days of 4 - 6 hours each [14]. According to the BAR-guidelines the multidisciplinary CR-team consisted of cardiologists, sports scientists and physiotherapists, psychologists, dietary specialists, nurses and social workers. All patients had a basic diagnostic evaluation including laboratory screening, echocardiography, ECG, and a stress test ideally performed at the beginning and at the end of the rehabilitation program. The CR centers furthermore provided an emergency room with full equipment, emergency laboratory tests, 24 hours ECG monitoring, 24 
hours blood pressure monitoring, duplex sonography of the vessels and psychological tests for evaluation of anxiety and depression.

The therapeutic program included endurance exercise training (ideally daily), dynamic strength training (two three times a week), physiotherapy, nursing, relaxation practices, psychological interventions. The rehabilitation program also included regular visits, professional advice and education with respect to physical exercise in daily practice, healthy nutrition, weight loss, stop smoking and reduction of psychological stress. German rehabilitation centers are regularly tested by the German pension funds whether the individual rehabilitation programs in each center meet their standards.

For routine data acquisition an electronic case report form was developed by a rehabilitation expert team in cooperation with the BNK Service GmbH, Munich, Germany, who also was responsible for the online service and data administration. All data have been anonymized. The case report included age, gender, the health care insurance responsible, diagnoses relevant for cardiac rehabilitation, social status, risk factors and risk diseases, body size, weight, body mass index, resting heart rate, blood pressure, laboratory parameters, medication, LV-function, maximal exercise performance. Data relevant for cardiovascular risk estimation were controlled at the end of the rehabilitation program. Furthermore the most important items for social reintegration and re-uptake of work were assessed.

Data evaluation was done by the Stiftung Institut für Herzinfarktforschung Ludwigshafen. The statistical analysis was descriptive focusing on the total population and data variations with respect to the participating rehabilitation centers, gender and age groups. For the statistical analysis continuous variables were expressed as means with standard deviations or medians with $25^{\text {th }}$ and $75^{\text {th }}$ percentiles, and were compared by using the Mann-Whitney-Wilcoxon test. Categorical variables are presented as absolute numbers and percentages, and were compared by using the chi-square test. The statistical comparisons were two-tailed, and p-values $<0.05$ were considered as statistically significant. All analyses were performed using SAS version 9.1 (SAS Institute Inc., Cary, NC, USA).

\section{Results}

Table 1 shows the distribution of the patients between the four participating rehabilitation centers. Almost all patients had a post-acute CR (98.7\%), with acute coronary syndrome or cardiovascular surgery as main reasons for CR admission. The population of the centers differed with respect to age and consequently with respect to the social status (e.g. employed or retired).

In Table 2 the clinical characteristics of the KARREE population are outlined. In addition to the total population three age groups (age $<50$ years, $50-70$ years, above 70 years) as well as gender differences were evaluated. Several clinical characteristics strongly depend on the particular age group including the CR initiating diagnosis. Although the majority of patients participated in CR after acute coronary syndrome, this diagnosis as the basis for CR-referral decreased significantly with age, whereas the number of patients after bypass surgery or aortic valve surgery increased. Also the cardiovascular risk factors in the patient's history varied with age. Whereas risk factors likephysical inactivity, overweight, psychosocial stress as well as cigarette smoking declined with age, diabetes and hypertension significantly increased (Table 2, Figure 1).

Table 1. Basic characteristics of participating ambulatory rehabilitation centers.

\begin{tabular}{|c|c|c|c|c|c|c|}
\hline & $\begin{array}{c}\text { Total } \\
\mathrm{n}=2989\end{array}$ & $\begin{array}{c}\text { Center (1) } \\
\mathrm{n}=988\end{array}$ & $\begin{array}{c}\text { Center (2) } \\
\mathrm{n}=1163\end{array}$ & $\begin{array}{c}\text { Center (3) } \\
\mathrm{n}=374\end{array}$ & $\begin{array}{c}\text { Center (4) } \\
\mathrm{n}=464\end{array}$ & p-value \\
\hline Female (\%) & 16.8 & 13.6 & 17.3 & 12.6 & 25.6 & $<0.0001$ \\
\hline Age (years) & $\begin{array}{c}65 \\
(55 / 73)\end{array}$ & $\begin{array}{c}61 \\
(53 / 70)\end{array}$ & $\begin{array}{c}64 \\
(54 / 72)\end{array}$ & $\begin{array}{c}62 \\
(53 / 72)\end{array}$ & $\begin{array}{c}73 \\
(68 / 77)\end{array}$ & $<0.0001$ \\
\hline Working (\%) & $\begin{array}{c}38.1 \\
(1089 / 2861)\end{array}$ & $\begin{array}{c}41,0 \\
(372 / 907)\end{array}$ & $\begin{array}{c}41.1 \\
(469 / 1141)\end{array}$ & $\begin{array}{c}45.6 \\
(166 / 364)\end{array}$ & $\begin{array}{c}18.3 \\
(82 / 449)\end{array}$ & $<0.0001$ \\
\hline Retired (\%) & $\begin{array}{c}53.3 \\
(1525 / 2861)\end{array}$ & $\begin{array}{c}46.1 \\
(418 / 907)\end{array}$ & $\begin{array}{c}51.2 \\
(584 / 1141)\end{array}$ & $\begin{array}{c}45.1 \\
(164 / 364)\end{array}$ & $\begin{array}{c}80.0 \\
(359 / 449)\end{array}$ & $<0.0001$ \\
\hline Unemployed (\%) & $\begin{array}{c}8.6 \\
(247 / 2861)\end{array}$ & $\begin{array}{c}12.9 \\
(117 / 907)\end{array}$ & $\begin{array}{c}7.7 \\
(88 / 1141)\end{array}$ & $\begin{array}{c}9.3 \\
(34 / 364)\end{array}$ & $\begin{array}{c}1.8 \\
(8 / 449)\end{array}$ & $<0.0001$ \\
\hline Post acute CR (\%) & 98.7 & 99.7 & 97.2 & 99.4 & 99.8 & \\
\hline
\end{tabular}

CR: cardiovascular rehabilitation; ns: not significant. 


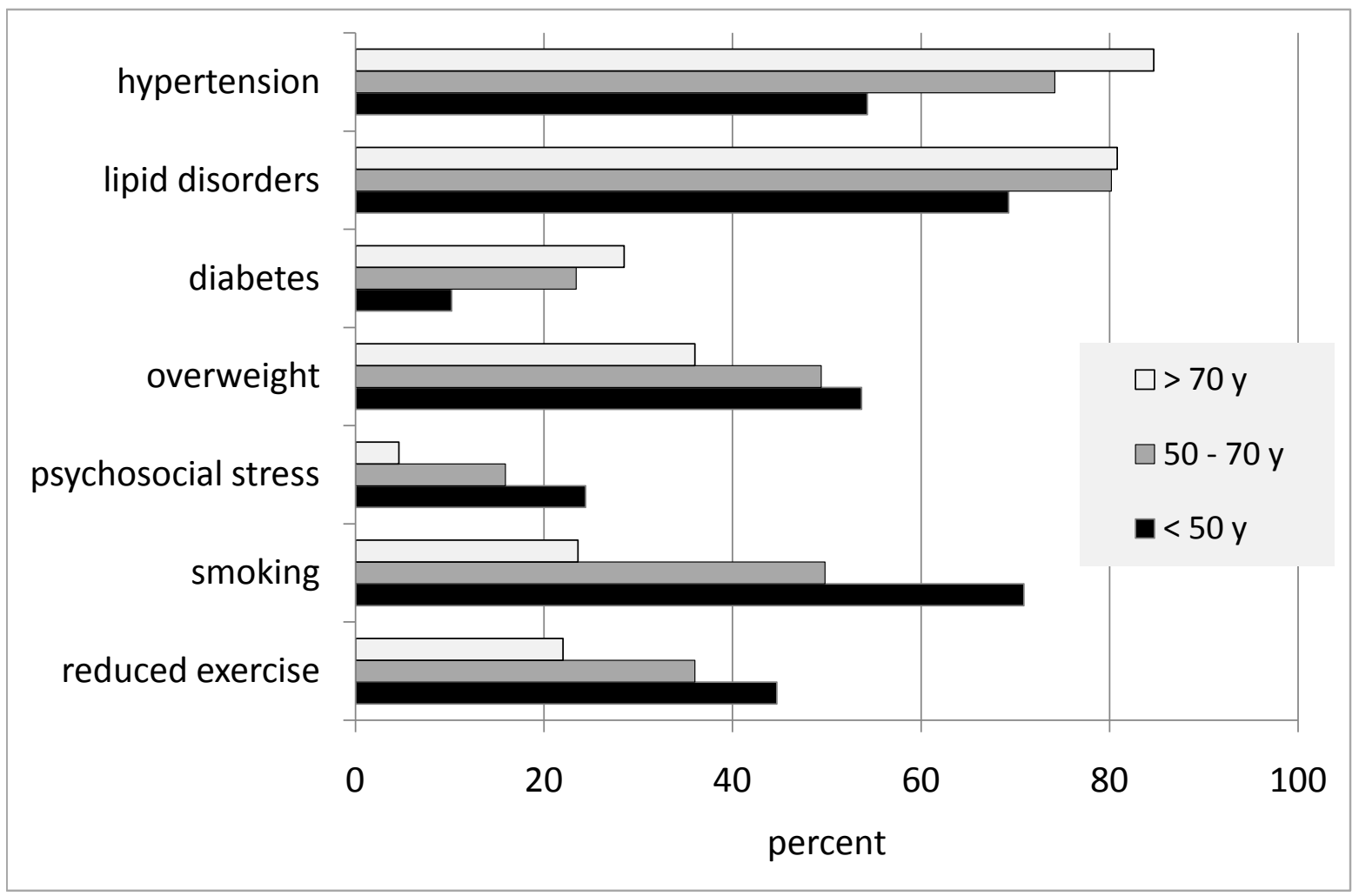

Figure 1. Age dependent distribution of various cardiovascular risk factors. Depending on the age group the prevalence of each of the selected risk factors varied significantly $(\mathrm{p}<0.0001)$.

At CR start major cardiovascular risk factors already were medically treated or positively influenced by individual behavior changes along the preceding in-hospital treatment. Thereby average LDL-cholesterol was measured only slightly above the target value of $100 \mathrm{mg} / \mathrm{dl}$, and triglycerides were fairly adjusted (Table 2). The average resting blood pressure also was within the expected target values.

Depression and anxiety only could be verified in a minority of the total population. However, both, depression and anxiety strongly depended on age, being highest in the young age group below 50 years. Furthermore, female patients were more prone to psychological stress (Table 2). These anamnestic reports could be confirmed by data from the HADS-tests showing the highest number of patients with pathological depression and anxiety scores in the young age group and in female gender (Table 2).

The majority of patients exhibited a normal or only slightly reduced left ventricular function. The stress test at the beginning of the rehabilitation showed a reasonable performance declining with age and being significantly lower in female patients. However, only $50.1 \%$ of all patients had a stress test at the CR start (Table 2).

Medical treatment of the patients reflected guideline recommendations already at CR start, and there were only slight changes in medication during the rehabilitation process (Table 3). In accordance with the prevalent risk diseases like diabetes and hypertension, drug prescription varied between the age groups. Especially the use of ARBs, vitamin-K antagonists, calcium channel blockers, diuretics and anti-diabetics increased with age.

During the course of the rehabilitation program there was a significant increase of exercise performance in all age groups independent of gender. In addition there was a further adjustment of other risk factors like a reduction of LDL-cholesterol and triglycerides. Also resting heart rate and blood pressure at rest were significantly reduced (Table 4).

From the patients supported by the German pension (DRV) funds $43.5 \%$ directly returned to work after rehabilitation. In addition $16.7 \%$ of the CR patients still working got support by organizing a stepwise reintegration into their previous working place. Almost all patients supported by the German pension funds were integrated into a special DRV aftercare program, whereas $65.5 \%$ of the retired patients were integrated into ambulatory heart groups. 
Table 2. Distribution of key characteristics between age groups and gender.

\begin{tabular}{|c|c|c|c|c|c|c|c|c|}
\hline & Total population & $<50$ years & 50 - 70 years & $>70$ years & p-value & Male & Female & $\mathrm{p}$-value \\
\hline $\begin{array}{c}\text { Number of patients } \\
\text { included }\end{array}$ & 2982 & $\begin{array}{l}17.9 \% \\
(535)\end{array}$ & $\begin{array}{l}56.2 \% \\
(1677)\end{array}$ & $\begin{array}{l}25.8 \% \\
(770)\end{array}$ & & $\begin{array}{c}83.3 \\
(2483)\end{array}$ & $\begin{array}{l}16.7 \\
(499)\end{array}$ & \\
\hline Female & $\begin{array}{c}16.7 \% \\
(499 / 2982)\end{array}$ & $\begin{array}{c}15.1 \% \\
(81 / 535)\end{array}$ & $\begin{array}{c}14.4 \% \\
(241 / 1677)\end{array}$ & $\begin{array}{c}23.0 \% \\
(177 / 770)\end{array}$ & $<0.0001$ & & & \\
\hline $\begin{array}{l}\text { Average age } \\
\text { (years) }\end{array}$ & 63 & 45 & 61 & 74 & $<0.0001$ & $\begin{array}{c}62 \\
(52-70)\end{array}$ & $\begin{array}{c}67 \\
(54-74)\end{array}$ & $<0.0001$ \\
\hline $\begin{array}{c}\text { Post-acute CR, AHB/AR } \\
\text { (\%) }\end{array}$ & 98.8 & 98.3 & 98.6 & 99.5 & & 98.7 & 98.8 & \\
\hline \multicolumn{9}{|l|}{ CR-initiation (\%) } \\
\hline Hospital & 66.6 & 75.1 & 68.1 & 57.0 & $<0.0001$ & 68.6 & 56.3 & $<0.0001$ \\
\hline GP & 0.9 & 1.3 & 0.8 & 0.9 & ns & 0.9 & 1.0 & ns \\
\hline Cardiologist & 11.8 & 2.1 & 9.1 & 24.4 & $<0.0001$ & 10.1 & 20.2 & $<0.0001$ \\
\hline Patients & 20.5 & 21.3 & 21.7 & 17.3 & $<0.05$ & 20.1 & 22.0 & Ns \\
\hline \multicolumn{9}{|l|}{ CR diagnoses (\%) } \\
\hline Post ACS & $59.9(1710 / 2854)$ & $70.9(360 / 508)$ & $60.3(970 / 1608)$ & $51.5(380 / 738)$ & $<0.0001$ & 59.2 & 63.5 & ns \\
\hline Post CABG & $17.1(489 / 2854)$ & $7.5(38 / 508)$ & $18.3(294 / 1608)$ & $21.3(157 / 738)$ & $<0.0001$ & 18.5 & 10.2 & $<0.0001$ \\
\hline Post valve repair & $8.4(241 / 2854)$ & $8.1(42 / 508)$ & $7.2(116 / 1608)$ & $11.3(84 / 738)$ & $<0.01$ & 8.5 & 8.4 & ns \\
\hline ICD & $0.9(25 / 2854)$ & $1.2(6 / 508)$ & $0.9(15 / 1608)$ & $0.5(4 / 738)$ & ns & 0.9 & 0.6 & ns \\
\hline PAD with vascular surgery & $1.2(33 / 2854)$ & $0.8(4 / 508)$ & $1.4(22 / 1608)$ & $0.9(7 / 738)$ & ns & 1.0 & 1.9 & ns \\
\hline \multicolumn{9}{|l|}{ History of risk factors: } \\
\hline Physical inactivity & $33.9(968 / 2854)$ & $44.7(227 / 508)$ & $36.0(579 / 1608)$ & $22.0(162 / 738)$ & $<0.0001$ & 34.0 & 33.4 & ns \\
\hline Diabetes & $22.4(639 / 2854)$ & $10.2(52 / 508)$ & $23.4(377 / 1608)$ & $28.5(210 / 738)$ & $<0.0001$ & 22.1 & 24.0 & ns \\
\hline Genetic disposition & $32.6(930 / 2854)$ & $38.2(194 / 508)$ & $32.3(520 / 1608)$ & $29.3(216 / 738)$ & $<0.01$ & 31.2 & 39.5 & $<0.001$ \\
\hline Lipid disorder & $78.4(2238 / 2854)$ & $69.2(352 / 508)$ & $80.2(1290 / 1608)$ & $80.8(596 / 738)$ & $<0.0001$ & 78.5 & 77.9 & ns \\
\hline Hypertension & $73.4(2094 / 2854)$ & $54.3(276 / 508)$ & $74.2(1193 / 1608)$ & $84.7(625 / 738)$ & $<0.0001$ & 72.7 & 76.8 & ns \\
\hline Smoking & $46.7(1334 / 2854)$ & $70.9(360 / 508)$ & $49.8(800 / 1608)$ & $23.6(174 / 738)$ & $<0.0001$ & 48.7 & 37.0 & $<0.0001$ \\
\hline Psychosocial stress & $14.5(413 / 2854)$ & $24.4(124 / 508)$ & $15.9(255 / 1608)$ & $4.6(34 / 738)$ & $<0.0001$ & 13.9 & 17.5 & $<0.05$ \\
\hline Overweight & $46.7(1333 / 2854)$ & $53.7(273 / 508)$ & $49.4(794 / 1608)$ & $36.0(266 / 738)$ & $<0.0001$ & 47.9 & 40.9 & $<0.01$ \\
\hline \multicolumn{9}{|l|}{$\begin{array}{c}\text { Risk factors as } \\
\text { evaluated at CR start: }\end{array}$} \\
\hline Fasting glucose & $111.7 \pm 43.0$ & $99.8 \pm 34.0$ & $113.6 \pm 43.2$ & $117.8 \pm 47.7$ & $<0.0001$ & $111.8 \pm 43.1$ & $111.0 \pm 42.4$ & ns \\
\hline Total cholesterol & $183.5 \pm 44.5$ & $190.1 \pm 44.4$ & $183.6 \pm 45.0$ & $178.8 \pm 43.1$ & $<0.0001$ & $181.0 \pm 43.8$ & $196.0 \pm 45.9$ & ns \\
\hline LDL cholesterol & $107.5 \pm 37.7$ & $112.7 \pm 36.1$ & $107.4 \pm 38.4$ & $104.3 \pm 36.8$ & $<0.0001$ & $106.3 \pm 37.2$ & $113.1 \pm 39.8$ & ns \\
\hline HDL cholesterol & $44.8 \pm 13.2$ & $44.1 \pm 14.4$ & $44.3 \pm 12.8$ & $46.3 \pm 13.1$ & $<0.0001$ & $43.2 \pm 11.8$ & $52.6 \pm 16.7$ & $<0.0001$ \\
\hline Triglycerides & $165.3 \pm 92.6$ & $177.5 \pm 100.9$ & $169.3 \pm 97.8$ & $148.0 \pm 70.0$ & $<0.0001$ & $166.8 \pm 95.4$ & $157.8 \pm 76.6$ & ns \\
\hline Systolic blood pressure & $127 \pm 20.4$ & $121.3 \pm 16.7$ & $127.9 \pm 20.4$ & $131.8 \pm 20.7$ & $<0.0001$ & $127.3 \pm 20.2$ & $130.1 \pm 21.9$ & $<0.05$ \\
\hline Diastolic blood pressure & $76.9 \pm 11.1$ & $77.5 \pm 11.2$ & $77.8 \pm 10.9$ & $74.5 \pm 11.1$ & $<0.0001$ & $76.9 \pm 11.1$ & $76.8 \pm 11.0$ & ns \\
\hline Actual smoking (\%) & $14.6(365 / 2498)$ & $28.7(140 / 487)$ & $14.4(209 / 1454)$ & $2.9(16 / 557)$ & $<0.0001$ & $14.8(313 / 2,111)$ & $13.4(52 / 387)$ & ns \\
\hline \multicolumn{9}{|l|}{ Depression at admission } \\
\hline$(\mathrm{HADS} \geq 11)(\%)$ & $9.2(166 / 1801)$ & $12.9(45 / 348)$ & $9.4(98 / 1043)$ & $5.6(23 / 410)$ & $<0.001$ & $8.7(130 / 1496)$ & $11.8(36 / 305)$ & 0.09 \\
\hline \multicolumn{9}{|l|}{ Anxiety at admission } \\
\hline$(\mathrm{HADS} \geq 11)(\%)$ & $14.0(253 / 1802)$ & $20.9(73 / 349)$ & $13.8(144 / 1,042)$ & $8.8(36 / 411)$ & $<0.0001$ & $12.6(189 / 1,505)$ & $21.8(66 / 303)$ & $<0.0001$ \\
\hline $\begin{array}{c}\mathrm{BMI} \\
\left(\mathrm{kg} / \mathrm{m}^{2}\right)\end{array}$ & $\begin{array}{c}28.4 \pm 4.6 \\
(97.9 \%)\end{array}$ & $\begin{array}{c}28.5 \pm 5.0 \\
(98.3 \%)\end{array}$ & $\begin{array}{c}28.7 \pm 4.8 \\
(98.5 \%)\end{array}$ & $\begin{array}{c}27.9 \pm 3.9 \\
(96.2 \%)\end{array}$ & $<0.05$ & $28.5 \pm 4.5$ & $28.1 \pm 5.4$ & $<0.05$ \\
\hline
\end{tabular}




\section{Continued}

\begin{tabular}{|c|c|c|c|c|c|c|c|c|}
\hline $\begin{array}{c}\text { Other clinical } \\
\text { characteristics as } \\
\text { evaluated at CR start: }\end{array}$ & & & & & & & & \\
\hline \multicolumn{9}{|l|}{ LV-function: } \\
\hline -Normal (\%) & $63.9(1775 / 2746)$ & $66.5(327 / 492)$ & $63.7(985 / 1546)$ & $62.6(443 / 708)$ & ns & $62.7(1437 / 2283)$ & $69.8(323 / 463)$ & $<0.01$ \\
\hline -Slightly reduced (\%) & $21.4(587 / 2746)$ & $21.1(104 / 492)$ & $20.8(322 / 1546)$ & $22.7(161 / 708)$ & ns & $22.2(507 / 2283)$ & $17.3(81 / 463)$ & $<0.05$ \\
\hline -Moderately reduced (\%) & $10.2(280 / 2746)$ & $8.3(41 / 492)$ & $10.7(165 / 1546)$ & $10.5(74 / 708)$ & ns & $10.5(239 / 2283)$ & $8.9(41 / 463)$ & ns \\
\hline -Severely reduced (\%) & $3.6(99 / 2746)$ & $3.7(18 / 492)$ & $4.1(63 / 1546)$ & $2.5(18 / 708)$ & ns & $3.7(85 / 2283)$ & $3.0(14 / 463)$ & ns \\
\hline \multicolumn{9}{|l|}{$\underline{\text { Stress test: }}$} \\
\hline Maximal performance (watt) & $96.4 \pm 38.3$ & $117.3 \pm 39.9$ & $96.2 \pm 36.2$ & $72.5 \pm 26.7$ & $<0.0001$ & $100.6 \pm 38.5$ & $69.6 \pm 23.9$ & $<0.0001$ \\
\hline (\% of total number evaluated) & 50.1 & 63.7 & 51.3 & 37.9 & & 52.0 & 40.7 & \\
\hline
\end{tabular}

AHB: Anschlussheilverfahren; AR: Anschlussrehabilitation; BMI: body mass index; CR: cardiovascular rehabilitation; ICD: implantable cardioverter defibrillator system; LV: left ventricular; ns: not significant.

Table 3. Medication at start and at the end of the CR-program.

\begin{tabular}{|c|c|c|}
\hline Pharmacological compounds & $\begin{array}{c}\text { Prescriptions at CR start } \\
\text { Number of evaluated patients } \\
\qquad \mathrm{n}=2842\end{array}$ & $\begin{array}{c}\text { Prescriptions at CR end } \\
\text { Number of evaluated patients } \\
\qquad \mathrm{n}=2842\end{array}$ \\
\hline $\begin{array}{c}\text { Beta-receptor blockers } \\
\text { (\% of total population; <50; } 50-70 ;>70 \text { years) }\end{array}$ & 87.3; 89.2; 87.1; 86.6 (ns) ${ }^{*}$ & 85.7; 87.6; 85.4; 85.1 (ns) ${ }^{*}$ \\
\hline $\begin{array}{c}\text { ACE-inhibitors } \\
\text { (\% of total population; <50; } 50-70 ;>70 \text { years) }\end{array}$ & $66.7 ; 69.3 ; 68.2 ; 61.6(<0.01)$ & $65.0 ; 66.5 ; 66.8 ; 60.2(<0.05)$ \\
\hline $\begin{array}{c}\text { ARBs } \\
\text { (\% of total population; }<50 ; 50-70 ;>70 \text { years) }\end{array}$ & $7.7 ; 3.5 ; 6.6 ; 13.0(<0.0001)$ & 8.8; 5.1; 7.4; $14.5(<0.0001)$ \\
\hline $\begin{array}{c}\text { Statins } \\
\text { (\% of total population; }<50 ; 50-70 ;>70 \text { years) }\end{array}$ & 79.1; 75.6; 81.3; 76.5 (ns) & 79.7; 75.6; 81.8; 78.1 (ns) \\
\hline $\begin{array}{c}\text { ASS } \\
\text { (\% of total population; }<50 ; 50-70 ;>70 \text { years) }\end{array}$ & 84.2; 82.7; 85.8; 81.8 (ns) & 82.2; 80.9; 83.7; 79.9 (ns) \\
\hline $\begin{array}{c}\text { Clopidogrel } \\
\text { (\% of total population; <50; } 50-70 ;>70 \text { years) }\end{array}$ & $56.1 ; 65.7 ; 56.7 ; 48.2(<0.0001)$ & $52.5 ; 60.4 ; 53.3 ; 45.2(<0.0001)$ \\
\hline $\begin{array}{c}\text { Vitamin-K antagonists } \\
\text { (\% of total population; <50; } 50-70 ;>70 \text { years) }\end{array}$ & $13.9 ; 11.6 ; 12.7 ; 18.3(<0.001)$ & $13.1 ; 11.0 ; 11.9 ; 17.3(<0.001)$ \\
\hline $\begin{array}{c}\text { Calcium channel blockers } \\
\text { (\% of total population; <50; } 50-70 ;>70 \text { years) }\end{array}$ & $10.1 ; 4.5 ; 9.7 ; 14.9(<0.0001)$ & $10.0 ; 3.7 ; 9.8 ; 14.9(<0.0001)$ \\
\hline $\begin{array}{c}\text { Diuretics } \\
\text { (\% of total population; }<50 ; 50-70 ;>70 \text { years) }\end{array}$ & $27.1 ; 13.4 ; 25.7 ; 39.5(<0.0001)$ & $27.6 ; 14.2 ; 26.9 ; 38.4(<0.0001)$ \\
\hline $\begin{array}{c}\text { Aldosterone antagonists } \\
\text { (\% of total population; <50; } 50-70 ;>70 \text { years) }\end{array}$ & $4.4 ; 6.7 ; 4.2 ; 3.3(<0.01)$ & $4.9 ; 6.9 ; 4.9 ; 3.7(<0.05)$ \\
\hline $\begin{array}{c}\text { Oral anti-diabetics } \\
\text { (\% of total population; < }<0 ; 50-70 ;>70 \text { years) }\end{array}$ & $11.3 ; 4.1 ; 11.8 ; 15.2(<0.0001)$ & $10.9 ; 4.1 ; 11.6 ; 14.2(<0.0001)$ \\
\hline $\begin{array}{c}\text { Insulin } \\
\text { (\% of total population; }<50 ; 50-70 ;>70 \text { years) }\end{array}$ & 5.8; 2.6; 5.9; $7.7(<0.0001)$ & 5.7; 2.6; 5.9; $7.7(<0.001)$ \\
\hline
\end{tabular}

ACE: Angiotensin-converting-enzyme; ARB: angiotensin-receptor-blocker; ASS: acetylic salicylic acid; ${ }^{\text {}) ~ s i g n i f i c a n c e ~ l e v e l s ~ r e f e r ~ t o ~ d i f f e r e n c e s ~ w i t h i n ~ t h e ~ a g e ~}$ groups. 
Table 4. Changes of risk parameters during rehabilitation.

\begin{tabular}{ccc}
\hline Parameter & Change (total population or as indicated) & p-value \\
\hline Body mass index $\left(\mathrm{kg} / \mathrm{m}^{2}\right)$ & decrease $0.1 \pm 0.7^{*}$ & $<0.0001$ \\
Total cholesterol $(\mathrm{mg} / \mathrm{dl})$ & decrease $16.7 \pm 35.8^{*}$ & $<0.0001$ \\
HDL-cholesterol $(\mathrm{mg} / \mathrm{dl})$ & increase $0.5 \pm 9.0^{*}$ & $<0.05$ \\
LDL-cholesterol $(\mathrm{mg} / \mathrm{dl})$ & Decrease $13.6 \pm 31.4^{*}$ & $<0.0001$ \\
Triglycerides $(\mathrm{mg} / \mathrm{dl})$ & Decrease $20.7 \pm 76.0^{*}$ & $<0.0001$ \\
Resting heart rate (beats/min) & Decrease $4.1 \pm 10.1^{*}$ & $<0.0001$ \\
Systolic blood pressure $(\mathrm{mmHg})$ & Decrease $5.9 \pm 16.9^{*}$ & $<0.0001$ \\
Diastolic blood pressure (mmHg) & Decrease $1.9 \pm 10.7^{*}$ & $<0.0001$ \\
Change in maximal performance in the stress test & increase $20.0 \pm 25.2$ (total group) \\
(watt) & increase $25.9 \pm 30.0(<50$ years) & increase $19.0 \pm 24.5(50-70$ years) \\
\hline
\end{tabular}

*no significant difference between the age groups.

\section{Discussion}

KARREE is the first registry evaluating ambulatory cardiovascular rehabilitation in Germany under the conditions of all day care and in alignment with the BAR-guidelines in a large cohort of patients treated in four rehabilitation centers. Although this registry only is an observational study there are several results of importance:

1) In the KARREE registry post-acute $\mathrm{CR}$ (AHB, Anschlussheilverfahren; AR, Anschlussrehabilitation) represented $98.7 \%$ of all patients (Table 1), whereas in the population of patients supported by the German pension funds (DRV) the proportion of post-acute CR only was 68\% in 2012 [19]. Therefore it may be suggested, that patients with chronic cardiovascular disease primarily are admitted to rehabilitation clinics. This potentially indicates a conflict of interest with respect to the distribution of the patients, as many rehabilitation clinics still are owned by the German pension funds, which may have influence on patients' allocation to various CR settings.

2) The proportion of patients with peripheral arterial disease (PAD) in this cohort is low. This potentially indicates a severe shortage of special care of this group of patients at least in some areas of Germany, and therefore deserves further investigation. As PAD patients especially benefit from cardiovascular rehabilitation by supervised implementation of systematic walking and support in smoking cessation, their admission and participation to CR should be clinical routine.

3) The baseline characteristics of the patients admitted to CR were similar as reported recently with an average age of 65 years in KARREE and 62 years of age in the OMEGA population (mostly in-patient rehabilitation) [7]. This clearly contradicts previous assumptions that ambulatory cardiovascular rehabilitation may be preferred by young patients [20]. Still, the proportion of female patients remains to be low raising the question of the attractiveness of ambulatory services for women.

4) All patients participating in KARREE reported a combination of several cardiovascular risks and risk diseases indicating a high risk population. However, depending on age a remarkable variation of the risk pattern could be observed. Whereas diabetes and hypertension were significantly increasing with age, smoking and psychosocial stress were decreasing. Although these data only have an observational basis, this age dependent risk pattern strongly indicates the necessity of an individual counselling and treatment of the patients during CR and thereafter. Especially in the young group of patients additional psychological support should be guaranteed in every rehabilitation center.

5) At least as far as cardiovascular risk factors can be treated by medication, this already has been consequently started during the patients' hospital stay. Therefore guideline adjusted medication primarily was continued during CR and changed only to a small extend. Moreover many recent smokers at least provisionally had 
stopped smoking already at rehabilitation start. Although during the rehabilitation program of three weeks the cardiovascular risk pattern still could be improved (Table 4) a major task of modern cardiovascular rehabilitation therefore is to further inform and educate the patients with respect to the individual relevance of cardiovascular prevention and to stimulate the individual motivation on compliance and adherence to preventive medication and life style. To improve sustainability of secondary prevention in the individual participation in after-care programs have been shown to be important [8] [21] [22]. It has to be noted that participation in such after-care programs could be achieved in the vast majority of these patients participating in ambulatory rehabilitation, and this is far above the numbers reported from inpatient cardiac rehabilitation [5].

6) Apart from long term implementation of cardiovascular prevention reintegration into normal life and especially into working ability is a major task of cardiovascular rehabilitation. Of the employed patients $43.5 \%$ could start working again directly after cardiac rehabilitation. In addition 16.75 were supported by stepwise reintegration into work. Unfortunately the register does not provide information on the remaining patients. This is a limitation of the registry, as especially blue color workers may keep serious limitations being a barrier against subsequent reintegration into work. This urgently needs to be investigated in more detail in future.

\section{Limitations of the Study}

This is an observational study reflecting patients characteristics and actual all day care in ambulatory CR in Germany. The data do not allow any suggestions on the clinical long-term effect of this intervention neither in comparison to inpatient CR nor in comparison to cardiac patients not participating CR. Moreover, for purposes of quality assurance the presented program of data acquisition and registration was too intensive and time consuming to be continuously integrated into all day care. Still, a simple and effective routine evaluation of the clinical short and long-term results by testing independent samples of patients participating and not participating $\mathrm{CR}$ are urgently needed for further development and improvement of this therapeutic instrument.

\section{Conclusion}

The KARREE registry reflects the actual clinical practice of ambulatory cardiac rehabilitation in Germany. Almost all patients consecutively enrolled in KARREE had an acute coronary syndrome or cardiovascular surgery before rehabilitation. This underscores the importance of a close networking between heart centers and cardiac rehabilitation facilities. Moreover, the majority of the patients were at high cardiovascular risk, and the marked differences of risk factor patterns between the age groups under investigation underscore the imperative necessity of an individualized therapeutic approach during cardiac rehabilitation.

\section{Acknowledgments}

This work is in remembrance of Doctor Gregor Sauer, who initiated and designed the registry in a leading position, and who prematurely paid the debt of nature. The registry was supported by the Deutsche Gesellschaft für Prävention \& Rehabilitation von Herz-Kreislauferkrankungen, e.V., DGPR.

\section{Conflicts of Interest}

From the participants of this study no conflicts of interest are reported.

\section{Ethical Considerations}

Within this survey data have consecutively and anonymously been collected within a program for quality assurance of the participating ambulatory rehabilitation centers. These centers were asked to strictly follow the current medical guidelines and the rules of good clinical practice. According to the German professional code of conduct a consultation of the local Ethics Committees is not required under these conditions.

\section{References}

[1] Korsukéwitz, Ch., Rohwetter, M. and Rauch, B. (2007) Definition und rechtliche Grundlagen der Rehabili-tation. In: 
Kardiologische Rehabilitation, Standards für die Praxis nach den Leitlinien der Deutschen Gesellschaft für Prävention und Rehabilitation von Herz-Kreislauferkrankungen e.V. (DGPR), Referenz-Reihe, Georg Thieme Verlag, 4-6, ISBN 978-3-13-143141-7.

[2] Korsukéwitz, C., Falk, J. and Lindow, B. (2012) Kardiologische Rehabilitation in Deutschland. Erfolgsmodell mit Perspektive: Herz, 37, 12-21.

[3] Willich, S.N., Müller-Nordhorn, J., Kulig, M., Binting, S., Gohlke, H., Hahmann, H., Bestehorn, K., Krobot, K. and Völler, H., PIN-Study Group. (2001) Cardiac Risk Factors, Medication, and Recurrent Clinical Events after Acute Coronary Disease. A Prospective Cohort Study. European Heart Journal, 22, 307-313. http://dx.doi.org/10.1053/euhj.2000.2294

[4] Bestehorn, K., Wegscheider, K. and Völler, H. (2008) Contemporary Trends in Cardiac Rehabilitation in Germany: Patient Characteristics, Drug Treatment, and Risk-Factor Management from 2000 to 2005. European Journal of Preventive Cardiology, 15, 312-318. http://dx.doi.org/10.1097/HJR.0b013e3282f40e14

[5] Jünger, C., Rauch, B., Schneider, S., Liebhart, N., Rauch, G., Senges, J. and Bestehorn, K. (2010) Effect of Early Short-Term Cardiac Rehabilitation after Acute ST-Elevation and Non-ST-Elevation Myocardial Infarction on 1-Year Mortality. Current Medical Research and Opinion, 26, 803-811. http://dx.doi.org/10.1185/03007991003604216

[6] Schwaab, B., Waldmann, A., Katalinic, A., Sheikhzadeh, A. and Raspe, H. (2011) In-Patient Cardiac Rehabilitation Versus Medical Care-A Prospective Multicentre Controlled 12 Months Follow-Up in Patients with Coronary Heart Disease. European Journal of Preventive Cardiology, 18, 581-586. http://dx.doi.org/10.1177/1741826710389392

[7] Rauch, B., Riemer, T., Schwaab, B., Schneider, S., Diller, F., Gohlke, H., Schiele, R., Katus, H., Gitt, A. and Senges, J., for the OMEGA Study Group. (2014) Short-Term Comprehensive Cardiac Rehabilitation after AMI Is Associated with Reduced 1-Year Mortality: Results from the OMEGA Study. European Journal of Preventive Cardiology, 21, 10601069. http://dx.doi.org/10.1177/2047487313486040

[8] Mayer-Berger, W., Simic, D., Mahmoodzad, J., Burtscher, R., Kohlmeyer, M., Schwitalla, B. and Redaèlli, M. (2014) Efficacy of a Long-Term Secondary Prevention Programme Following Inpatient Cardiovascular Rehabilitation on Risk and Health-Related Quality of Life in a Low-Education Cohort: A Randomized Controlled Study. European Journal of Preventive Cardiology, 21, 145-152. http://dx.doi.org/10.1177/2047487312465526

[9] Salzwedel, A., Nosper, M., Röhrig, B., Linck-Eleftheriadis, S., Strandt, G. and Völler, H. (2014) Outcome Quality of In-Patient Cardiac Rehabilitation in Elderly Patients-Identification of Relevant Parameters. European Journal of Preventive Cardiology, 21, 172-180. http://dx.doi.org/10.1177/2047487312469475

[10] Bjarnason-Wehrens, B., Predel, H.G., Graf, C. and Rost, R. (1999) Ambulante kardiale Rehabilitation der Phase II„Kölner Modell“—einschließlich der Ergebnisse drei Jahre nach Abschluss der Rehabilitation. Herz, 24, 9-23. http://dx.doi.org/10.1007/BF03042127

[11] Gysan, D., Heinzler, R. and Schmidt, K. (1999) Auswirkungen einer vierwöchigen ambulanten kardialen Rehabilitationsmaßnahme (Phase II) auf kardiovaskuläre Risikofaktoren, körperliche Belastbarkeit und berufliche Reintegration bei Patienten nach Myokardinfarkt, Dilatationsbehandlung und Herzoperation. Herz, 24, 44-56. http://dx.doi.org/10.1007/BF03042131

[12] Wendt, T. (1999) Ambulante Phase II Rehabilitation Herzkranker an einem im Ballungsraum Rhein-Main gelegenen Akutkrankenhaus: Das Frankfurter Modell. Herz, 24, 57-62. http://dx.doi.org/10.1007/BF03042132

[13] Bjarnason-Wehrens, B., Bott, D., Benesch, L., Bischoff, K.O., Buran-Kilian, B., Gysan, D., Hollenstein, U., Mayer-Berger, W., Wilkniss, R. and Sauer, G. (2007) Long-Term Results of a Three-Week Intensive Cardiac Out-Patient Rehabilitation Program in Motivated Patients with Low Social Status. Clinical Research in Cardiology, 96, 77-85. http://dx.doi.org/10.1007/s00392-007-0461-0

[14] Bundesarbeitsgemeinschaft für Rehabilitation , BAR (2005) Rahmenempfehlungen zur ambulanten kardiologischen Rehabilitation. Frankfurt am Main, Dezember 2005.

[15] Badura, B., Grande, G., Janßen, H. and Schott, T. (1994) Evaluation kardiologischer Rehabilitation-ein Vergleich von Struktur-, Prozess- und Ergebnisqualität stationärer und ambulanter Anschlussheilbehandlung. In: Die Betriebskrankenkasse; Zeitschr. des Bundesverbandes der Betriebskrankenkassen, Essen, 500-517.

[16] Iseringhausen, O., Schott, T. and vom Order, A. (2002) Die Qualität der Organisation Kardiologischer Rehabilitation-Ein Vergleich stationärer und ambulanter Versorgungsformen. Rehabilitation, 41, 130-139. http://dx.doi.org/10.1055/s-2002-28447

[17] Schweikert, B., Hahmann, H., Steinacker, J.M., et al. (2009) Intervention Study Shows Outpatient Cardiac Rehabilitation to Be Economically at Least as Attractive as Inpatient Rehabilitation. Clinical Research in Cardiology, 98, 787795. http://dx.doi.org/10.1007/s00392-009-0081-6

[18] Steinacker, J.M., Li, Y., Muche, R., Koenig, W., Hahmann, H., Imhof, A., Kropf, C., Brandstetter, S., Schweikert, B., Leidl, R. and Schiefer, D.H. (2011) Long Term Effects of Comprehensive Cardiac Rehabilitation in an Inpatient and 
Outpatient Setting. Swiss Medical Weekly, 140, Article ID: w13141.

[19] Völler, H. (2013) Rehabilitation. In: Deutscher Herzbericht 2013, Deutsche Herzstiftung (Hrsg.), Deutsche Herzstiftung e.V., Frankfurt am Main, 180-185, ISBN 978-3-9811926-6-7.

[20] Karoff, M., Müller-Fahrnow, W., Kittel, J., Vetter, H.O., Gülker, H. and Spyra, C. (2002) Outpatient Cardiological Rehabilitation-Acceptance and Conditions Related to Choice of Setting. Die Rehabilitation, 41, 167-174. http://dx.doi.org/10.1055/s-2002-28441

[21] Buchwalsky, G., Buchwalsky, R. and Held, K. (2002) Langzeitwirkungen der Nachsorge in einer ambulanten Herzgruppe. Eine Fall-Kontrollstudie. Zeitschrift für Kardiologie, 91, 139-146. http://dx.doi.org/10.1007/s003920200003

[22] Giannuzzi, P., Temporelli, L., Marchioli, R., Maggioni, A., Balestroni, G., Ceci, V., Chieffo, C., Gattone, M., Griffo, R., Schweiger, C., Tavazzi, L., Urbinati, S., Valagussa, F. and Vanuzzo, D., GOSPEL Investigators (2008) Globalsecondary Prevention Strategies to Limit Event Recurrence after Myocardial Infarction. JAMA Internal Medicine, 168, 2194-2204. http://dx.doi.org/10.1001/archinte.168.20.2194 
Scientific Research Publishing (SCIRP) is one of the largest Open Access journal publishers. It is currently publishing more than 200 open access, online, peer-reviewed journals covering a wide range of academic disciplines. SCIRP serves the worldwide academic communities and contributes to the progress and application of science with its publication.

Other selected journals from SCIRP are listed as below. Submit your manuscript to us via either submit@scirp.org or Online Submission Portal.
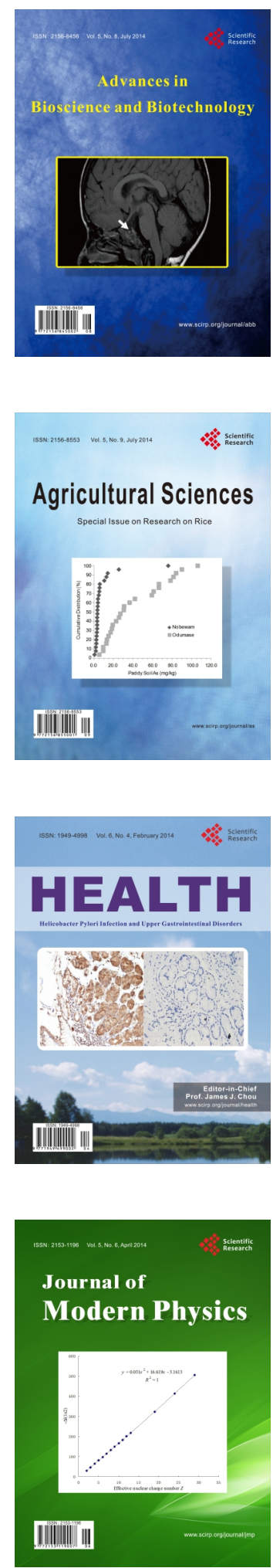
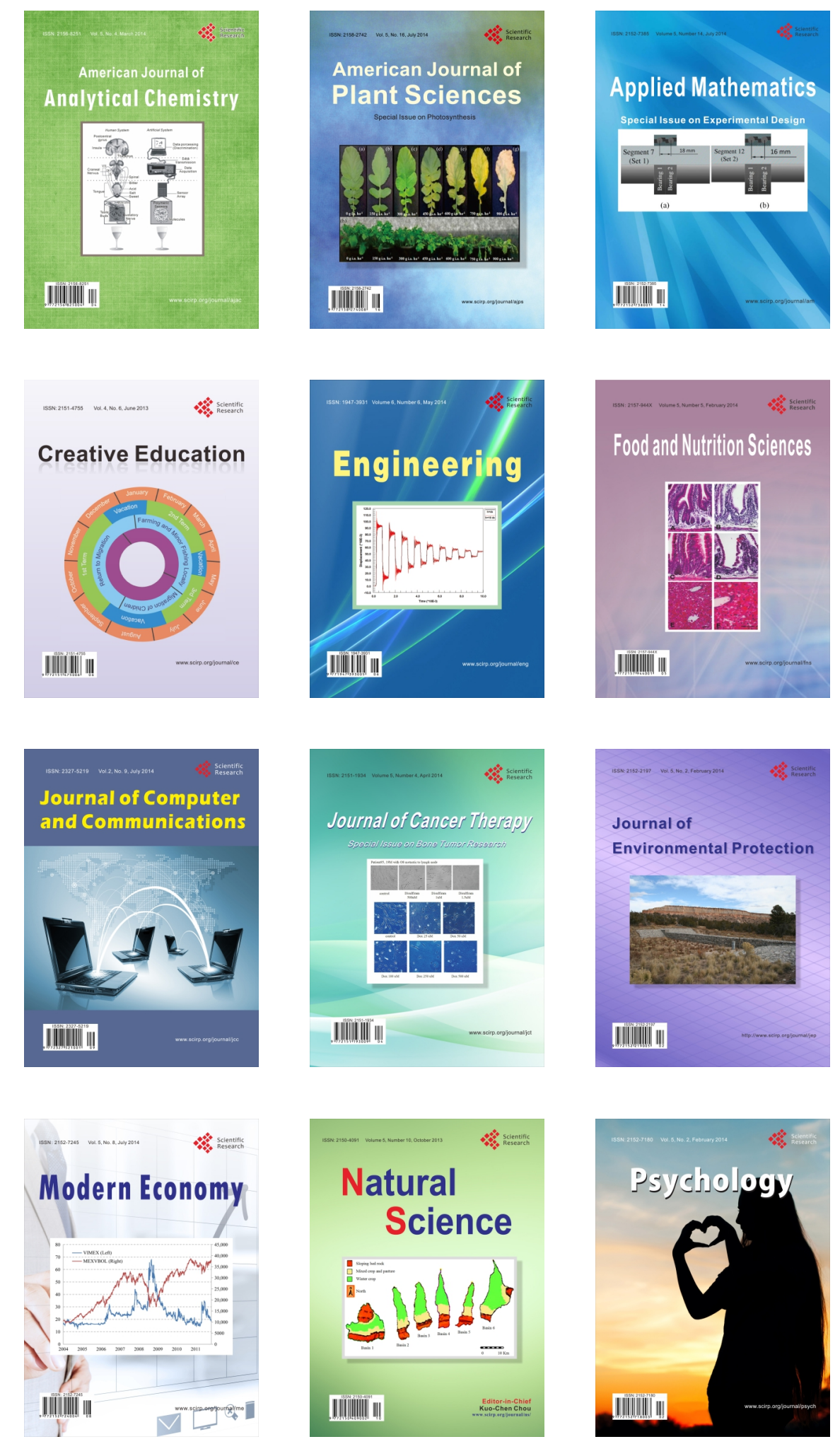